Pacific Journal of Mathematic 


\title{
ON COMPLETELY HAUSDORFF-COMPLETION OF A COMPLETELY HAUSDORFF SPACE
}

\author{
ASIT BARAN RAHA
}

\begin{abstract}
R. M. Stephenson, Jr. (Trans. Amer. Math. Soc. 133 (1968), 537-546) has established the existence of a completely Hausdorff-closed extension $X^{\prime}$ of an arbitrary completely Hausdorff space $X$. Stephenson demonstrates that $X^{\prime}$ enjoys many interesting properties of the Stone-Čech compactification. This paper shows that, by a modification of the topology, $X^{\prime}$ is made also to possess a property which is in the line of the celebrated property of the Stone-Čech compactification of a completely regular Hausdorff space that it is the largest amongst all Hausdorff compactifications.
\end{abstract}

1. Introduction. A topological space $X$ is called completely Hausdorff if for every pair $x, y$ of distinct points of $X$ there exists a continuous real valued function $f$ on $X$ such that $f(x) \neq f(y)$. A completely Hausdorff space is called completely Hausdorff-closed if every homeomorphic image of it in any completely Hausdorff space is closed. A space $Y$ is termed a completely Hausdorff-closed extension of a completely Hausdorff space $X$ if $X$ is dense in $Y$ and $Y$ is completely Hausdorff-closed. R. M. Stephenson, Jr. in [4] has established the existence of a completely Hausdorff-closed extension (referred to as the completely Hausdorff-completion) $X^{\prime}$ of an arbitrary completely Hausdorff space $X$. If $X$ is completely regular (which, of course, assumes Hausdorff property and is necessarily completely Hausdorff) then $X^{\prime}$ is the Stone-Cech compactification of $X$. Stephenson shows $\{[4]$, Theorem 4$\}$ that, even if $X$ is completely Hausdorff but not necessarily completely regular, $X^{\prime}$ continues to enjoy many interesting properties of the Stone-Čech compactification. By enlarging the topology of $X^{\prime}$ we shall, in fact, strengthen Theorem 4 of [4] in the sense that property (vii) therein will be replaced by the following:

$X^{\prime}$ is a projective maximum in the class of completely Hausdorffclosed extensions $Y$ of $X$ with the property that any element in $F(X)$, the set of all continuous functions on $X$ into $[0,1]$, admits an extension to $F(Y)$.

The above property is, obviously, akin to the well-known fact that the Stone-Cech compactification is largest among the Hausdorff compactifications of a completely regular Hausdorff space.

2. Notations and definitions. We shall try to follow the notations and definitions of [4] as far as possible. 
$C(X)$ will stand for the set of all bounded continuous functions on $X$. If $Z$ is any topological space, we shall denote by $C(X, Z)$ the set of all continuous mappings of $X$ into $Z$.

A topological space $Y$ is an extension space of another space $X$ if $X$ is dense in $Y$. If $T$ is an extension space of a topological space $S$, the tracefilter's of $T$ are the filters $\mathscr{T}(t), t \in T-S$, where $T(t)$ is the filter on $S$ given by $\{U \cap S: U$ a neighbourhood of $t$ in $T$.

Banaschewski [1] introduced the notion of a projective maximum in a set $E$ of extensions of $X$; an extension $Y$ in $E$ is a projective maximum in $E$ if for each $Z$ in $E$ there is a continuous function from $Y$ onto $Z$ which leaves $X$ pointwise fixed.

A filter $F$ on a space $X$ is called completely regular provided that it has a base ${ }^{3}$ of open sets such that for each $B \in \mathbb{S}^{3}$, there is a set $B^{\prime} \subset B$ in $\mathscr{S}$ and a function $f \in F(X)$ such that $f\left(B^{\prime}\right)=0$ and $f=1$ on $X-B$.

3. Main result. Let $X$ be a completely Hausdorff space, and let $/ l$ be the set of all maximal completely regular filters on $X$ which have empty adherences. (If $\mathscr{F}$ is a completely regular filter,

$$
\cap\{F: F \in \mathscr{F}\}=\cap\{\bar{F}: F \in \mathscr{F}\}=\text { adherence of } \mathscr{F} \text {, }
$$

where $\bar{F}=$ closure of $F$ in $X$. If $\cap\{F: F \in \mathscr{F}\}=\varnothing, \mathscr{F}$ is called free, otherwise it is called fixed.) Put $X^{\prime}=X \cup-\not l$. We shall endow $X^{\prime}$ with a topology as follows:

Any set, open in $X$, is also open in $X^{\prime}$. If $\mathscr{F} \in \mathscr{l}$, basic neighbourhoods of $\mathscr{F}$ are of the form $G \cup\{\mathscr{F}\}$ where $G \in \mathscr{F}$. With this topology (will, henceforth, be called the Katétov topology) $X^{\prime}$ becomes a completely Hausdorff-closed space and will be called the completely Hausdorff-completion of $X$. The trace filters of $X^{\prime}$ are the filters $\{\mathscr{T}(\mathscr{F}): \mathscr{F} \in \mathscr{C}\}$ and for each $\mathscr{F} \in \mathscr{C l}, \mathscr{T}(\mathscr{F})=\left\{U \cap X: U \subset X^{\prime}\right.$ and $U$ a neighbourhood of $\mathscr{F}\}=\{G: G \in \mathscr{F}\}=\mathscr{F}$. Thus the trace filters of $X^{\prime}$ are the maximal completely regular filters $\mathscr{F}$ on $X$ such that

$$
\cap\{G: G \in \mathscr{F}\}=\varnothing \text {. }
$$

Now we are in a position to state our main theorem which is identical with Theorem 4 of [4] with the exception of property (vii).

THeOREM 1. Let $X$ be a completely Hausdorff space. The completely Hausdorff-completion $X^{\prime}$ of $X$ has the following properties:

(i) If $Z$ is a compact Hausdorff space, then each function in $C(X, Z)$ has a unique extension in $C\left(X^{\prime}, Z\right)$.

(ii) The Stone-Weierstrass theorem holds for $X^{\prime}$. 
(iii) $X^{\prime}$ is locally connected if and only if $X$ is locally connected and each trace filter of $X^{\prime}$ has a base consisting of connected open sets.

(iv) $X^{\prime}$ is locally connected only if $X$ is locally connected and pseudocompact.

(v) $X^{\prime}$ is connected if and only if $X$ is connected.

(vi) $C\left(X^{\prime}\right)$ and $C(X)$ are isomorphic, and if $R$ is the real line, $C\left(X^{\prime}\right)$ and $C(X, R)$ are isomorphic only if $X$ is pseudocompact.

(vii) Suppose $Y$ is a completely Hausdorff-closed space containing $X$ as a dense subset and each element of $F(X)$ has an extension to $F(Y)$. Then there exists a one-to-one function $g \in C\left(X^{\prime}, Y\right)$ such that $g\left(X^{\prime}\right)=Y$ and $g$ is identity on $X$. In short, $X^{\prime}$ is a projective maximum in the class of completely Hausdorff-closed extensions $Y$ of $X$ with the property that any element in $F(X)$ admits an extension to $F(Y)$.

Proof. Proofs of (i) - (vi) are omitted as they are same as those given in Theorem 4 of [4] (page 540). We shall only give a proof for (vii). Let $Y$ be a completely Hausdorff-closed topological space containing $X$ as a dense subset and such that every function in $F(X)$ admits an (unique) extension to $F(Y)$. If $\mathscr{F}$ is a nonconvergent maximal completely regular filter on $X$ (i.e., $\mathscr{F} \in \mathscr{C}$ ) define $Z=$ $\left\{f \in F(X)\right.$ : for some $G^{\prime}, G \in \mathscr{F}$ with $G^{\prime} \subset G$, one has $f\left(G^{\prime}\right)=0$ and $f(X-G)=1\} . Z$ is nonvoid as $\mathscr{F}$ is completely regular. For $f \in F(X)$ let $f^{\prime}$ denote its extension in $F(Y)$. Put $Z^{\prime}=\left\{f^{\prime}: f \in Z\right\}$. Take $\mathscr{S}=$ $\left\{V\left(f^{\prime}, t\right)=f^{\prime-1}[0, t): f^{\prime} \in Z^{\prime}, 0<t \leqq 1\right\}$. The empty set does not belong to $\mathscr{S}$. Consider, $V\left(f_{i}^{\prime},{ }_{i}\right) \in \mathscr{S}, i=1,2, \cdots, n$ and choose, for each $i, 0<s_{i}<t_{i}$. By using the normality of $[0,1]$ we can get $g_{i} \in F(Y)$ such that $g_{i}\left(V\left(f_{i}^{\prime}, s_{i}\right)\right)=0$ and $g_{i}\left[Y-V\left(f_{i}^{\prime}, t_{i}\right)\right]=1$ for $i=1,2, \cdots, n$. Put $g=\max _{1 \leqq i \leqq n} g_{i}$. Then $g \in F(Y)$ and $g\left[\bigcap_{i=1}^{n} V\left(f_{i}^{\prime}, s_{i}\right)\right]=0$ and

$$
g\left[Y-\bigcap_{j=1}^{n} V\left(f_{j}^{\prime}, t_{j}\right)\right]=1 .
$$

Note also that $\bigcap_{j=1}^{n} V\left(f_{j}{ }^{\prime}, s_{j}\right) \subset \bigcap_{j=1}^{n} V\left(f_{j}{ }^{\prime}, t_{j}\right)$. Thus, we have shown that finite intersections of sets of $\mathscr{S}$ form a completely regular filter base on $Y$. Let $\mathscr{G}$ be the completely regular filter on $Y$ generated by $\mathscr{S}$ and let $\mathscr{U}$ denote a maximal completely regular filter on $Y$ such that $\mathscr{C} \subset \mathscr{Q}$. Since $Y$ is completely Hausdorff-closed every completely regular filter on $Y$ has nonempty adherence (See [4] Theorem 1, and [2]). Consequently adherence of $\mathscr{C}(=a d(\mathscr{U}))$ is nonempty and maximality of $\mathscr{C}$ will make $\mathscr{C}$ converge to each point in $\operatorname{ad}(\mathscr{U})$. But $Y$ is Hausdorff, so $\operatorname{ad}(\mathscr{C})$ must contain exactly one point, i.e., $\cap U=$ $\cap\{U: U \in \mathscr{C}\}$ is a singleton. We now claim that $\mathscr{F}=\{U \cap X: U \in \mathscr{Q}\}$.

Proof of the claim. Since $\mathscr{C}$ is a maximal completely regular 
open filter it has a completely regular filter base $\mathscr{Y}$ consisting of open sets. As $X$ is dense in $Y$, it is easy to see that $26 \cap X=$ $\{U \cap X: U \in \mathscr{Z}\}$ is an open filter on $X$ with an open base given by $\mathscr{Y} \cap X=\left\{V \cap X: V \in \mathscr{V}^{-}\right\}$. Let $V \cap X \in \mathscr{Y}^{\circ} \cap X$. Since $V \in \mathscr{Y}$ there exist $V^{\prime} \in y^{\prime}$ with $V^{\prime} \subset V$ and $h \in F(Y)$ such that $h\left(V^{\prime}\right)=0$ and $h(Y-V)=1$. Obviously, $h\left(V^{\prime} \cap X\right)=0$ and $h(X-V \cap X)=1$. Let $f$ denote the restriction of $h$ to $X$. Then $f \in F(X)$ and $f\left(V^{\prime} \cap X\right)=0$ and $f(X-V \cap X)=1$ i.e., $\mathscr{Y} \cap X$ is a completely regular filter base on $X$ for $\mathscr{E} \cap X$. Therefore $\mathscr{E} \cap X$ is a completely regular filter on $X$. Again $\mathscr{F}$ is a completely regular filter on $X$, so $F \in \mathscr{F}$ implies that there exist $F^{\prime} \in \mathscr{F}$ with $F^{\prime} \subset F$ and $f \in F(X)$ such that $f\left(F^{\prime}\right)=0$ and $f(X-F)=1$. This gives $F^{\prime} \subset f^{-1}[0,1) \subset F$. Hence $f \in Z$ and $F^{\prime} \subset f^{\prime-1}[0,1) \cap X \subset F$ where $f^{\prime} \in Z^{\prime}$. Now, $f^{\prime-1}$ $[0,1) \in \mathscr{C} \subset$. Thus $X \cap f^{\prime-1}[0,1) \in \mathbb{C} \cap X$ and $F \supset X \cap f^{\prime-1}[0,1)$ implies $F \in \mathscr{C} \cap X$ (since it is a filter). We get $\mathscr{F} \subset \mathscr{C} \cap X$ and maximality of $\mathscr{F}$ forces $\mathscr{F}=\mathscr{E} \cap X$. Immediately we have from the above fact, $(\cap U) \cap X=\cap(U \cap X)=\cap\{F: F \in \mathscr{F}\}=\varnothing$ as $\mathscr{F}$ is a free maximal completely regular filter. So the single point contained in $\cap U$ is actually in $Y-X$. Let the point be denoted by $y(\mathscr{F})$. Next we show that if $\mathscr{F}_{1}$ and $\mathscr{F}_{2}$ are two distinct points in $\mathscr{C}$, the points $y\left(\mathscr{F}_{1}\right)$ and $y\left(\mathscr{F}_{2}\right)$ are distinct points of $Y-X$. Since $\mathscr{F}_{1}$ and $\mathscr{F}_{2}$ are two distinct free maximal completely regular filters there must exist $G_{1} \in \mathscr{F}_{1}$ and $G_{2} \in \mathscr{F}_{2}$ such that $G_{1}$ and $G_{2}$ are open in $X$ and $G_{1} \cap G_{2}=\varnothing$. As shown earlier, we can associate two maximal completely regular filters $\mathscr{C}_{1}$ and $\mathscr{C}_{2}$ on $Y$ with $\mathscr{F}_{1}$ and $\mathscr{F}_{2}$ respectively. By definition $\left\{y\left(\boldsymbol{F}_{i}\right)\right\}=\cap\left\{U: U \in \mathscr{C}_{i}\right\}, i=1,2$ and we also know that $\mathscr{F}_{i}=\mathscr{C}_{i} \cap X$. Consequently there exists $U_{i} \in \mathscr{C}_{i}$ such that $U_{i} \cap X=G_{i}$ and $U_{i}$ is open $(i=1,2)$. Since $G_{1} \cap G_{2}=\varnothing$ and $X$ is dense in $Y$ we have $U_{1} \cap U_{2}=\varnothing$. Since $y\left(\mathscr{F}_{i}\right) \in \mathscr{Z}_{i}$ for $i=1,2$ we get $y\left(\mathscr{F}_{1}\right) \neq y\left(\mathscr{F}_{2}\right)$. So far we have shown that $\mathscr{F} \mapsto y(\mathscr{F})$ is a one-to-one map of $\mathscr{L}$ into $Y-X$. Let $i$ denote the identity map on $X$ into $Y$.

Define $\bar{i}: X^{\prime} \rightarrow Y$ as follows:

$$
\begin{array}{lr}
\bar{i}(x)=i(x)=x & \text { if } x \in X, \text { and } \\
\bar{i}(\mathscr{F})=y(\mathscr{F}) & \text { if } \mathscr{F} \in \mathscr{C}=X^{\prime}-X .
\end{array}
$$

Claim: $\bar{i}$ is continuous.

We shall establish the continuity by showing the continuity at each point.

(i) Suppose $x \in X$. Then $\bar{i}(x)=x$. Let $W$ be an open neighbourhood of $x$ in $Y$, then $\bar{i}^{-1}(W) \cap X=i^{-1}(W)=G$, an open neighbourhood of $x$ in $X$ and hence open in $X^{\prime}$ and also $\bar{i}(G) \subset W$.

(ii) For $\mathscr{F} \in \mathscr{C}$, we have $\bar{i}(\mathscr{F})=y(\mathscr{F})$. By construction of 
$y(\mathscr{F})$ we know that it is the point of convergence of a maximal completely regular filter $\mathscr{U}$ on $Y$ such that $\mathscr{F}=\mathscr{C} \cap X$.

If $W$ is an open neighbourhood of $y(\mathscr{F})$ in $Y$ then $W \in \mathscr{Q} 6$ i.e., $W \cap X \in \mathscr{F}$. But $W \cap X$ is open in $X$ and hence $(W \cap X) \cup\{\mathscr{F}\}$ is an open neighbourhood of $\mathscr{F}$ in $X^{\prime}$ such that

$$
\begin{aligned}
\bar{i}[(W \cap X) \cup\{\mathscr{F}\}] & =\bar{i}(W \cap X) \cup \bar{i}(\mathscr{F})=i(W \cap X) \cup\{y(\mathscr{F})\} \\
& =(W \cap X) \cup\{y(\mathscr{F})\} \subset W .
\end{aligned}
$$

Thus the continuity of $\bar{i}$ has been proved. But $X^{\prime}$ is, in particular, completely Hausdorff-closed and $\bar{i}$ is a continuous function on $X^{\prime}$ into a completely Hausdorff space $Y$ in which $X$ is dense. Consequently, from the following fact it will follow that $\bar{i}$ is onto $Y$.

Fact. Let $X$ be a completely Hausdorff-closed space and let $Y$ be a completely Hausdorff space such that there is a continuous function $f: X \rightarrow Y$. Then $f(X)$ is a completely Hausdorff closed subspace of $Y$.

Let us put $g=\bar{i}$. Then $g \in C\left(X^{\prime}, Y\right)$ with $g\left(X^{\prime}\right)=Y$ and $g$ restricted to $X$ equals $i$, the identity map on $X$.

Corollary 1. Suppose $Y$ is completely Hausdorff-closed space satisfying the conditions stated in Theorem 1(vii) and $f$ is a homeomorphism of $X$ onto $X$, then there exists a one-to-one function $g \in C\left(X^{\prime}, Y\right)$ such that $g\left(X^{\prime}\right)=Y$ and $g$ restricted to $X$ equals $f$.

Proof. We first note that if $\mathscr{F}$ is a nonconvergent maximal completely regular filter on $X, f(\mathscr{F})$ is a nonconvergent maximal completely regular filter on $X$. Then the proof follows by a reasoning similar to one presented in the proof of Theorem 1(vii) where $i$ is replaced by $f$.

4. REMARKS. The completely Hausdorff-completion $X^{\prime}$ of $X$ in Theorem 1 is essentially unique, i.e., if $T$ is any completely Hausdorff closed extension of $X$ and $T$ satisfies the properties of Theorem 1 then $X^{\prime}$ and $T$ are homeomorphic. For there exists $g \in C\left(X^{\prime}, T\right)$ such that $g\left(X^{\prime}\right)=T$ and $g$ is identity on $X$. Also, there exists $h \in C\left(T, X^{\prime}\right)$ such that $h(T)=X^{\prime}$ and $h$ is identity on $X$. Therefore by the following result $\left\{[3]\right.$, page 5\} we can assert that $X^{\prime}$ and $T$ are homeomorphic.

Result. Let $X$ be dense in each of the Hausdorff spaces $S$ and $T$. If the identity mapping on $X$ has continuous extensions $s$ from 
$S$ into $T$, and $t$ from $T$ into $S$, then $s$ is a homeomorphism onto, and $s^{-1}=t$.

One can raise the following two questions regarding Theorem 1: (a) Is a $Y$ satisfying the condition (vii) of Theorem 1 homeomorphic to $X^{\prime}$ ? (b) Is $X^{\prime}$ a one-to-one continuous image of such $Y$ ? We shall answer both the questions in the negative. Let $N$ denote the set of natural numbers with discrete topology. On $N$ any free maximal completely regular filter is nothing but a free ultrafilter. Thus $\beta N=N U, \not C$ where $\mathscr{C}$ is the set of all free ultrafilters on $N$. The topology by which $\beta N$ is the Stone-Cech compactification of $N$ will be called Stone-Cech topology $(S-\check{C}$ topology) for $\beta N$. Its open sets are generated by $\left\{V^{\prime}: V\right.$ open in $\left.N\right\}$ where $V^{\prime}=V \cup\{\mathscr{F} \in \mathscr{C l}: V \in \mathscr{F}\}$. But, according to our definition, $\beta N$ endowed with the Katertov topology is the completely Hausdorff-completion of $N$ and in this topology $\mathscr{l}=\beta N-N$ is a closed, discrete infinite subspace of $\beta N$ and, thus, cannot be compact. While in the $S-\check{C}$ topology of $\beta N$, $\mathscr{C}$ is closed, no doubt, and hence compact. Clearly, the $S-\breve{C}$ topology is strictly weaker than the Katětov topology. As $S-\breve{C}$ topology of $\beta N$ is compact, no continuous map from $\beta N$ with $S-\check{C}$ topology onto $\beta N$ with Katétov topology can exist. So homeomorphism is ruled out. But the Stone-Cech compactification $\beta N$ satisfies all the conditions enjoyed by a $Y$ in Theorem 1(vii).

Acknowledgement. The author expresses his thanks to Dr. Ashok Maitra for suggesting some modifications to an earlier version of the paper and especially for raising the questions discussed under the caption "Remarks".

\section{REFERENCES}

1. B. Banaschewski, Extensions of topological spaces, Canad. Math. Bull., 7 (1965), 1-22.

2. - On the Weierstrass-Stone approximation theorem, Fund. Math., 44 (1957), 249-252.

3. L. Gillman and M. Jerison, Rings of continuous functions, Van Nostrand, Princeton, N. J., 1960.

4. R. M. Stephenson, Jr., Spaces for which the Stone-Weierstrass theorem holds, Trans. Amer. Math. Soc., 133 (1968), 537-546.

Received November 16, 1970.

Indian Statistical Institute, Calcutta 


\section{PACIFIC JOURNAL OF MATHEMATICS}

\section{EDITORS}

H. SAMELSON

Stanford University

Stanford, California 94305

C. R. Hовву

University of Washington

Seattle, Washington 98105
J. DUGUNDJI

Department of Mathematics

University of Southern California

Los Angeles, California 90007

RICHARD ARENS

University of California

Los Angeles, California 90024

\section{ASSOCIATE EDITORS}
E. F. BECKENBACH
B. H. NeumanN
F. WOLF
K. YoSHIDA

\section{SUPPORTING INSTITUTIONS}

UNIVERSITY OF BRITISH COLUMBIA

CALIFORNIA INSTITUTE OF TECHNOLOGY

UNIVERSITY OF CALIFORNIA

MONTANA STATE UNIVERSITY

UNIVERSITY OF NEVADA

NEW MEXICO STATE UNIVERSITY

OREGON STATE UNIVERSITY

UNIVERSITY OF OREGON

OSAKA UNIVERSITY

UNIVERSITY OF SOUTHERN CALIFORNIA
STANFORD UNIVERSITY

UNIVERSITY OF TOKYO

UNIVERSITY OF UTAH

WASHINGTON STATE UNIVERSITY

UNIVERSITY OF WASHINGTON

AMERICAN MATHEMATICAL SOCIETY CHEVRON RESEARCH CORPORATION NAVAL WEAPONS CENTER 


\section{Pacific Journal of Mathematics}

\section{Vol. 38, No. $1 \quad$ March, 1971}

Bruce Alan Barnes, Banach algebras which are ideals in a Banach algebra ..... 1

David W. Boyd, Inequalities for positive integral operators............... 9

Lawrence Gerald Brown, Note on the open mapping theorem .............. 25

Stephen Daniel Comer, Representations by algebras of sections over Boolean

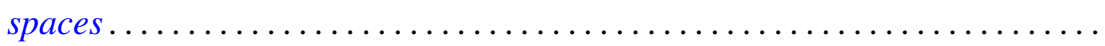

John R. Edwards and Stanley G. Wayment, On the nonequivalence of

conservative Hausdorff methods and Hausdorff moment sequences ........

P. D. T. A. Elliott, On the limiting distribution of additive functions $(\bmod 1) \ldots \ldots$

Mary Rodriguez Embry, Classifying special operators by means of subsets

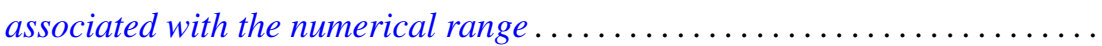

Darald Joe Hartfiel, Counterexamples to a conjecture of G. N. de Oliveira ......

C. Ward Henson, A family of countable homogeneous graphs...............

Satoru Igari and Shigehiko Kuratsubo, A sufficient condition for

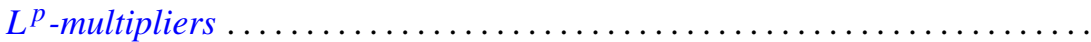

William A. Kirk, Fixed point theorems for nonlinear nonexpansive and

generalized contraction mappings............................

Erwin Kleinfeld, A generalization of commutative and associative rings ...... 95

D. B. Lahiri, Some restricted partition functions. Congruences modulo $11 \ldots \ldots 103$

T. Y. Lin, Homological algebra of stable homotopy ring $\pi *$ of spheres ....... 117

Morris Marden, A representation for the logarithmic derivative of a meromorphic function...........................

John Charles Nichols and James C. Smith, Examples concerning sum properties for metric-dependent dimension functions . .

Asit Baran Raha, On completely Hausdorff-completion of a completely

Hausdorff space.

M. Rajagopalan and Bertram Manuel Schreiber, Ergodic automorphisms and affine transformations of locally compact groups..........

N. V. Rao and Ashoke Kumar Roy, Linear isometries of some function

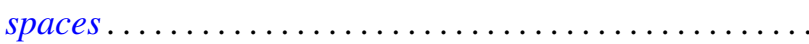

William Francis Reynolds, Blocks and F-class algebras of finite groups

Richard Rochberg, Which linear maps of the disk algebra are multiplicative ...

Gary Sampson, Sharp estimates of convolution transforms in terms of decreasing

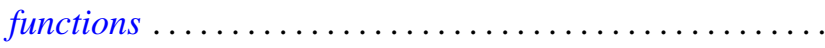

Stephen Scheinberg, Fatou's lemma in normed linear spaces

Ken Shaw, Whittaker constants for entire functions of several complex

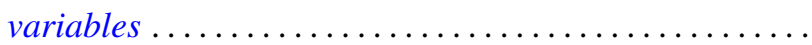

James DeWitt Stein, Two uniform boundedness theorems................ 251

$\mathrm{Li} \mathrm{Pi} \mathrm{Su,} \mathrm{Homomorphisms} \mathrm{of} \mathrm{near-rings} \mathrm{of} \mathrm{continuous} \mathrm{functions} \mathrm{.} \mathrm{.............} 261$

Stephen Willard, Functionally compact spaces, $C$-compact spaces and mappings of minimal Hausdorff spaces....................... 